\title{
Factores asociados al síndrome de desgaste laboral en auxiliares de enfermería
}

\author{
Factors associated with burnout syndrome in nursing assistants
}

\author{
Gustavo Bergonzoli1* orcid.org/0000-0001-9728-5329 \\ Carolina Jaramillo-López' ${ }^{1}$ orcid.org/0000-0002-4096-6518 \\ David Delgado-Grijalba² orcid.org/0000-0002-0803-9060 \\ Felipe Duque ${ }^{3}$ orcid.org/0000-0002-1924-0085 \\ Germán Vivas 3 orcid.org/0000-0003-4214-3635 \\ Felipe José Tinoco-Zapata ${ }^{4}$ orcid.org/0000-0002-4096-6518
}

1. Grupo de Gestión del Conocimiento e Innovación (GECOI) del Hospital Tomas Uribe Uribe. Tuluá, Valle.

2. $\quad$ Subproceso de Investigación, Hospital Tomas Uribe Uribe. Tuluá, Valle.

3. Subproceso de Salud Familiar y Comunitaria, Hospital Tomas Uribe Uribe. Tuluá, Valle.

4. Gerente del Hospital Tomas Uribe Uribe. Tuluá, Valle.

\section{Resumen}

Introducción: El Síndrome de Desgaste Laboral o Burnout, es entendido como una baja resiliencia para afrontar el estrés laboral. Objetivo: Identificar factores asociados al Burnout y la prevalencia global y de cada uno de sus constructos, entre auxiliares de enfermería en un hospital de referencia de mediana y alta complejidad, en Colombia. Materiales y métodos: Estudio de casos y controles, con 183 auxiliares registradas en un hospital. Se utilizó el instrumento de Maslach y se identificaron los factores asociados utilizando la regresión Log-Binomial determinando los Odds Ratio (OR). Resultados: Los factores encontrados asociados con Burnout que aumentan la probabilidad de ocurrencia son: trabajar más de 48 horas por semana, OR=1,02 (1,01-1.05) al 90\% de confianza; sentirse mal con el trabajo que se realiza $\mathrm{OR}=3,87$ (1,18-10,71); el dormir siete horas o más al día OR=0,70 $(0,53-0,91)$, disminuye la probabilidad. La prevalencia total de Burnout fue 44,7\%, siendo 11,8\% para agotamiento personal, 14,9\% despersonalización y 47,81\% pobre realización personal. Conclusiones: Los factores asociados: no dormir suficiente y sentirse mal con la tarea que se realiza, aumentan la probabilidad de desarrollar Burnout, mientras que dormir 7 o más horas diarias la disminuye. Estos hallazgos facilitarán la aplicación de medidas de control institucional.

Palabras clave: Agotamiento emocional; auxiliares de enfermería; estudios de casos y controles; regresión logística. (Fuente: DeCS, Bireme).

\begin{abstract}
Introduction: The Burnout syndrome is understood as a low resilience to face work stress. Objective: To identify factors and constructs associated with Burnout and its overall prevalence in nursing assistants in a reference hospital of medium and high complexity level from Colombia. Materials and methods: A case-control study was conducted on 183 nursing assistants from a hospital. The Maslach instrument and Log-Binomial regression were used to identify associated factors, determining their Odds Ratio (OR). Results: The factors found associated with Burnout that increase its probability of occurrence are: working more than 48 hours a week (OR=1.02 (1.01-1.05) 90\% CI) and feeling negatively about the work being done (OR=3.87 (1.18-10.71)). However, sleeping 7 or more hours a day reduces such probability $(\mathrm{OR}=0.70(0.53-0.91))$. The total Burnout prevalence was $44.7 \%$, the main causes being personal fatigue (11.8\%), depersonalization (14.9\%), and poor personal fulfillment (47.81\%). Conclusions: Associated factors, such as not getting enough sleep and feeling negatively about the work being done, increase the probability to develop Burnout, while this probability decreases by sleeping 7 or more hours a day. These findings will facilitate the implementation of institutional control measures.
\end{abstract}

Keywords: Burnout, psychological; nursing assistants; case-control studies; logistic models. (Fuente: DeCS, Bireme). 


\section{Introducción}

El lugar de trabajo es considerado, por la Organización Internacional del Trabajo (OIT)(1), un determinante muy importante para la salud. Por lo tanto, este debe ser fuente de salud y bienestar y no de enfermedad. Sin embargo, no siempre es así y algunas veces, se convierte en un lugar que genera malestar y mala salud.

Según Bailey(2) el Síndrome de Desgaste Laboral (SDL), también conocido como Burnout en inglés, se considera como una respuesta inadecuada a las demandas laborales derivadas de la atención a personas, por eso ha sido estudiado preferentemente, en los trabajadores del sector sanitario; debido a que su ocurrencia está asociada a un desequilibrio entre las aspiraciones y competencias personales, y las exigencias laborales institucionales. El trastorno psicológico se desarrolla de forma lenta y progresiva $\mathrm{y}$, por tanto, puede pasar inadvertido durante mucho tiempo por la persona afectada. Los efectos de dicho desequilibrio se encuentran mediados por las estrategias de afrontamiento personales y la habilidad para manejar los factores estresantes del entorno laboral. Se considera el SDL como una combinación de signos y síntomas típicos de estrés psicológico y no como un diagnóstico clínico. El SDL tiene un impacto negativo en el desempeño de los individuos.

Recientemente, en Estados Unidos, la creciente evidencia ha despejado dudas sobre lo que está ocurriendo con los médicos de ese país, ya que más del 50\% de ellos están "quemados" por el trabajo clínico según Nguyen et al.,(3); es claro que se está frente a una situación epidémica.

El agotamiento físico y el estrés entre las enfermeras es común, el trabajo de enfermería suele ser una ocupación estresante. La prevalencia del agotamiento en la enfermería es un problema real y una amenaza para el sistema de atención de la salud. Altos niveles del SDL, entre el personal de enfermería, han sido reportados en Europa, Asia y América del Norte. Un estudio transversal multicéntrico, que involucró 23.159 funcionarias del área de enfermería hospitalaria, reportó altos niveles del síndrome de desgaste laboral, así, Inglaterra 42\%, Irlanda 41\%, Polonia 40\%, Alemania 30\%, España 29\%, Bélgica $25 \%$, Noruega $24 \%$, Finlandia 22\%, Suiza $15 \%$ y Holanda $10 \%(4,5)$.
Las causas del agotamiento no sólo son inherentes a las personas, sino también a factores atribuibles a la gestión y organización del trabajo. Los factores organizacionales como el trabajo por turnos, la sobrecarga de trabajo, la claridad de rol y la ambigüedad de las funciones, son factores asociados con el SDL en el personal de enfermería. Según el estudio de Vargas et al.,(6) la posibilidad de padecer el SDL se aumenta en un $23 \%$ en las enfermeras por cada paciente adicional en su carga de trabajo. La relación de una enfermera a cinco pacientes es considerada aceptable, pero cuando esta se incrementa, se asocia con el síndrome, y más del $40 \%$ de las enfermeras de los hospitales suelen tener una relación de uno a ocho pacientes.

Muchas enfermeras deben trabajar horas extra de manera obligatoria, sin tener la opción de evitar esta situación. El SDL afecta al $49 \%$ de las enfermeras menores de 30 años y al $40 \%$ de las mayores. La repercusión económica de dichas afecciones es alta, como se puede deducir de los datos sobre el ausentismo laboral y la renovación del personal. Más del $10 \%$ de las reclamaciones con respecto a enfermedades laborales se atribuye al estrés en el trabajo(7).

El SDL ha sido exhaustivamente estudiado en médicos y enfermeras profesionales, pero muy poco en otros miembros del equipo de salud, como las auxiliares de enfermería. El objetivo de este estudio fue estimar la prevalencia global para cada constructo e identificar los factores asociados al Burnout, entre auxiliares de enfermería en un hospital de referencia de mediana y alta complejidad, en Colombia.

\section{Materiales y métodos}

Estudio observacional de casos y controles para identificar los factores asociados y estimar la prevalencia del SDL entre las auxiliares de enfermería vinculadas a una institución de referencia de mediana y alta complejidad del Valle del Cauca, Colombia.

En la décima Clasificación Internacional de Enfermedades (CIE-10) de la Organización Mundial de la Salud (OMS), definió al SDL como "un estado de agotamiento vital". En la CIE-11, publicada en mayo de 2018, el SDL aparece con el código QD85 y se describe como "un proceso en el que las tensiones y ansiedades cotidianas van minando gradualmente la 
salud mental y física de una persona"(8). La CIE-11 está iniciando su proceso de implementación en Colombia.

En los criterios de inclusión se consideraron auxiliares de enfermería que desempeñaban una actividad asistencial en la institución durante el periodo de estudio y con un mínimo de seis meses de antigüedad, independientemente del tipo de contratación y que aceptaran participar después de haber leído y firmado el consentimiento informado. Se excluyeron del estudio a auxiliares con antecedentes personales de o con trastornos mentales actuales.

\section{Población de estudio}

De acuerdo a los registros de la oficina de Talento Humano de la institución, ésta contaba con 190 auxiliares de enfermería vinculadas en el momento de la encuesta. De ellas, 4 tenían menos de 6 meses de antigüedad en la institución, 3 se desvincularon antes de la realización de la encuesta y 2 rehusaron participar, el porcentaje de participación fue del $84,7 \%$.

El puntaje para clasificar a los sujetos como padeciendo o no el SDL, se estimó sacando el promedio de los valores arrojados por los tres componentes definidos para la caracterización del SDL: Agotamiento Emocional (AE), Despersonalización (DP) y Realización Personal (RP). Este puntaje se consideró de acuerdo con lo propuesto en estudios anteriores (9-12), tal como se relaciona en la Tabla 1 . Una vez obtenido el puntaje individual, se ordenó la serie, se calculó la mediana y se clasificó a las auxiliares con puntaje igual o superior a la mediana como casos, mientras que las que obtuvieron un puntaje inferior se consideraron como controles ${ }^{(9-12)}$. Los estadísticos descriptivos de la frecuencia estimada, fueron: promedio $=46,8$, mediana $=47,0, \quad \operatorname{moda}=48,0 \quad$ y desviación estándar $= \pm 11,4$.

Tabla 1. Puntaje para clasificación de los sujetos en estudio (9-12)

\begin{tabular}{lrrr}
\hline $\begin{array}{c}\text { Nivel } \\
\text { del } \\
\text { SDL }\end{array}$ & $\begin{array}{c}\text { Agotamiento } \\
\text { emocional }\end{array}$ & Despersonalización & $\begin{array}{c}\text { Realización } \\
\text { personal }\end{array}$ \\
\hline Bajo & $=<18$ & $=<5$ & $=>40$ \\
Medio & $19-26$ & $6-9$ & -39 \\
Alto & $=>27$ & $=>10$ & $=<33$ \\
\hline
\end{tabular}

Definición de caso. Auxiliares de enfermería cuya suma de los puntajes medio y alto para AE y DP, más los puntajes de baja y mediana RP; fueran igual o superior al valor de la mediana, cuyo valor se estableció en 47, resultando 81 auxiliares, 79 mujeres y 2 hombres.

Definición de control. Auxiliares de enfermería cuyo puntaje estuviera por debajo de la mediana, resultando 80 auxiliares, 74 mujeres y 6 hombres.

\section{Recolección y control de calidad de la información}

Los datos fueron obtenidos mediante la aplicación de la versión en español del cuestionario del Maslach(11), instrumento diseñado para medir el SDL y validado en varios contextos latinoamericanos, incluido el colombiano como el realizado en la Universidad del Valle en Cali(12). Se recolectaron datos complementarios para medir variables potencialmente confusoras.

La recolección de los datos fue realizada por encuestadoras capacitadas, quienes entrevistaron a las auxiliares de enfermería participantes del estudio. Para asegurar la calidad en la recolección de los datos, las encuestas diligenciadas eran revisadas diariamente y semanalmente el $10 \%$ de las encuestas digitadas seleccionadas al azar. La información fue procesada y analizada con el software estadístico SPSS versión $24^{\circledR(13) .}$

\section{Plan de análisis}

Análisis exploratorio. En esta fase, cada variable fue examinada para evaluar la normalidad de su distribución e identificar valores extremos que pudieran afectar la estabilidad de los estadísticos estimados (medidas de asociación). Se evaluó la presencia de errores numéricos tales como la colinealidad, establecida mediante el estadístico Factor de Inflación de la Varianza (FIV), la presencia de celdas con cero y la existencia de completa separación. Durante esta fase se estimó la prevalencia total y para cada uno de los tres componentes principales del SDL.

Análisis multivariado. Se realizó una selección de variables utilizando el procedimiento estadístico de eliminación paso a paso (Stepwise procedure). Para la identificación de los factores potencialmente asociados con el SDL y dado que la variable respuesta estaba medida en una escala nominal dicotómica, se utilizó la regresión log-binomial. Se utilizó el Odds Ratio (OR) para estimar la fuerza de la asociación entre la variable respuesta $y$ las variables 
independientes de interés en el estudio, ajustando por una serie de covariables que pudieran fungir como potenciales variables de confusión. La media de asociación OR se estimó tomando el antilogaritmo de cada uno de los coeficientes betas de las variables independientes arrojados por el modelo, con su respectivo intervalo al 95\% de confianza. Se realizó el diagnóstico del modelo obtenido, para esto se evaluó la bondad de ajuste utilizando la prueba de HosmerLemeshow ${ }^{(14,15)}$.

\section{Consideraciones éticas}

El protocolo fue sometido para revisión y aprobación por el Comité de Evaluación Ética y Científica de la
Investigación en seres humanos o con muestras de origen humano (CEI), de la ESE Hospital Departamental Tomás Uribe Uribe de Tuluá-Empresa Social del Estado (HDTUU), según registrado en el acta No. 8, del 13 de septiembre de 2017.

\section{Resultados}

Durante el análisis exploratorio bivariado realizado con la finalidad de caracterizar los casos y controles, se encontró diferencia estadísticamente significativa en algunas de las variables en estudio (Tabla 2).

Tabla 2. Caracterización de los Sujetos en estudio

\begin{tabular}{|c|c|c|c|}
\hline Variable & $\begin{array}{c}\text { Caso } \\
\text { n (\%) }\end{array}$ & $\begin{array}{c}\text { Control } \\
\text { n (\%) }\end{array}$ & Estadístico \\
\hline \multicolumn{4}{|l|}{ Sexo } \\
\hline Mujeres & $79(97,5)$ & $74(92,5)$ & $\mathrm{Chi}^{2}=2,16, \mathrm{gl}=1, p=0,14$ \\
\hline Hombres & $2(2,5)$ & $6(7,5)$ & \\
\hline Total & $81(100)$ & $80(100)$ & \\
\hline \multicolumn{4}{|l|}{ Estado civil } \\
\hline Soltero & $21(27,3)$ & $33(42,9)$ & $\mathrm{Chi}^{2}=10,9, \mathrm{gl}=3, p=0,01$ \\
\hline Unión Libre & $21(27,3)$ & $26(33,8)$ & \\
\hline Casado & $17(22,1)$ & $12(15,6)$ & \\
\hline Separado & $13(16,9)$ & $4(5,2)$ & \\
\hline Viudo & $5(6,4)$ & $2(2,5)$ & \\
\hline Total & 77 (100) & $77(100)$ & \\
\hline Religión (Si) & $64(85,3)$ & $69(90,8)$ & $\mathrm{Chi}^{2}=1,98, \mathrm{gl}=1, p=0,16$ \\
\hline \multicolumn{4}{|l|}{ Raza } \\
\hline Blanca & $18(26,5)$ & $21(28)$ & \\
\hline Mestiza & $47(69,1)$ & $50(66,7)$ & $\mathrm{Chi}^{2}=0,09, \mathrm{gl}=1, p=0,77$ \\
\hline Afro & $3(4,4)$ & $4(5,3)$ & \\
\hline Total & $68(100)$ & $75(100)$ & \\
\hline Consume licor & 27 & 20 & $\mathrm{Chi}^{2}=1,37, \mathrm{gl}=1, p=0,24$ \\
\hline Uso de tabaco & 1 & 0 & Fisher $=1=0,50$ \\
\hline Sentirse mal desde ingreso & 16 & 3 & $\mathrm{Chi}^{2}=10,3, \mathrm{gl}=1, p=0,001^{*}$ \\
\hline Sentirse mal actualmente & 25 & 8 & $\mathrm{Chi}^{2}=11,0, \mathrm{gl}=1, p=0,001^{*}$ \\
\hline Edad & 39,2 & 30,6 & $\mathrm{t}=1,9, \mathrm{gl}=148, p=0,06$ \\
\hline Talla & 1,62 & 1,61 & $\mathrm{t}=-3,57, \mathrm{gl}=146, p=0,72$ \\
\hline Peso & 67,4 & 67,8 & $\mathrm{t}=0,20, \mathrm{gl}=152, p=0,85$ \\
\hline Años de trabajo & 6,1 & 9,8 & $\mathrm{t}=-2,1, \mathrm{gl}=84, p=0,04^{*}$ \\
\hline Horas trabajadas mes & 53,9 & 47 & $\mathrm{t}=2,01, \mathrm{gl}=148, p=0,04^{*}$ \\
\hline Horas de sueño día & 6,6 & 7,4 & $\mathrm{t}=-3,41, \mathrm{gl}=143, p=0,001^{*}$ \\
\hline
\end{tabular}

El análisis exploratorio mostró una distribución cuadrática en la relación entre la edad y los años de trabajo en la institución, en las auxiliares de enfermería que fueron clasificadas con el SDL. Los grupos etarios estudiados arrojaron los siguientes resultados, de 19 a 29 años, 2 casos (34,6\%); de 30 a 39,12 casos (14,8\%); de 40 a 49, $15(18,5 \%)$ y de 50 y más, 20 casos $(24,7 \%)$. El análisis para la función cuadrática arrojó los siguientes resultados, ANOVA, $\mathrm{F}=6,92, \mathrm{gl}=1,42 ; p=0,01$. Prueba de homogeneidad de las varianzas, Levene $=4,14$, gl 3,42; $p=0,01$ (Figura 1).
Un hallazgo novedoso, por lo inesperado, fue la distribución bimodal del SDL, lo cual sugiere que la presencia de dos diferentes grupos de factores asociados con el SDL, unos que tienen mayor impacto en los grupos jóvenes, entre los cuales podrían mencionarse la inexperiencia laboral o la falta de competencias para afrontar situaciones laborales difíciles; mientras que a edades mayores pueden estar impactando factores como el desgaste físico y mental acumulado a lo largo de los años de trabajo. 
Esta relación cuadrática no fue encontrada entre las auxiliares sin SDL.

La prevalencia global del síndrome del desgaste por el trabajo, entre las auxiliares que consintieron participar en el estudio, fue de 44,7\% (72/161), fundamentalmente a expensas de la baja realización personal, cifra considerada alta si se compara con otros estudios similares. Se estimó la prevalencia global y para cada uno de los componentes principales del síndrome del desgaste por el trabajo (Tabla 3).

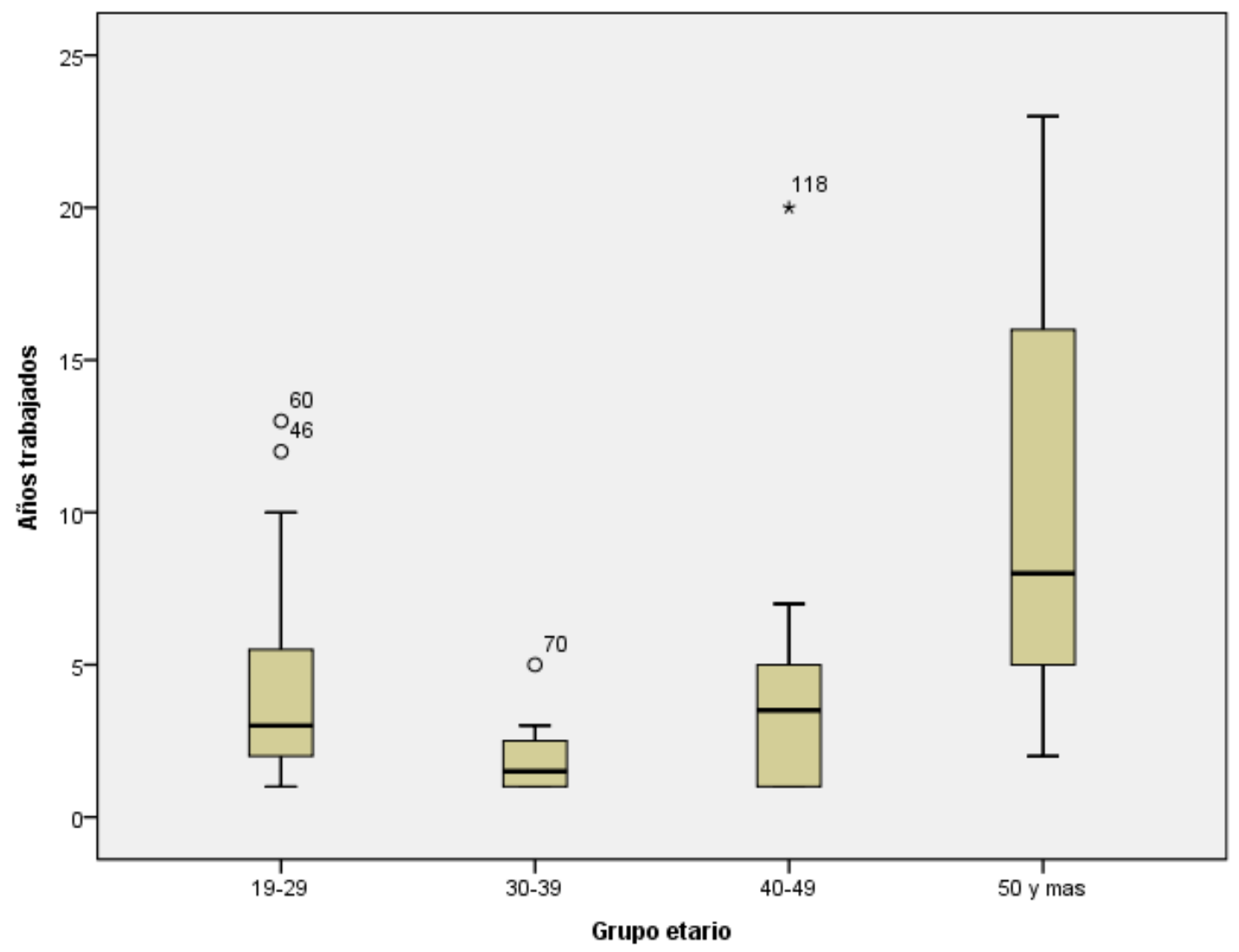

Figura 1. Distribución del SDL en Auxiliares de enfermería, según edad

Tabla 3. Prevalencia del SDL total y para cada uno de sus constructos

\begin{tabular}{lcccl}
\hline \multicolumn{1}{c}{ Constructo } & $\begin{array}{c}\text { Alto } \\
\text { n (\%) }\end{array}$ & $\begin{array}{l}\text { Medio } \\
\text { n (\%) }\end{array}$ & $\begin{array}{c}\text { Bajo } \\
\text { n (\%) }\end{array}$ & $\begin{array}{l}\text { Total } \\
\text { n (\%) }\end{array}$ \\
\hline Agotamiento Emocional & $7 / 161(4,4)$ & $12 / 161(7,5)$ & $142 / 161(88,2)$ & $19 / 161(11,8)$ \\
Despersonalización & $7 / 161(4,4)$ & $17 / 161(10,6)$ & $134 / 161(83,2)$ & $24 / 161(14,9)$ \\
Realización Personal & $46 / 161(28,6)$ & $31 / 161(19,3)$ & $84 / 161(52,2)$ & $77 / 161(47,8)$ \\
\hline
\end{tabular}

La información contenida en la (Tabla 1), sirvió de base para la identificación de las variables que serían incluidas en el análisis multivariado, para evaluar si la desigual distribución de las variables, entre los casos y controles, representaba una potencial variable de confusión o un factor de riesgo real. Algunas variables sin significancia estadística fueron tenidas en cuenta en el análisis multivariado debido a su importancia epidemiológica o por haber sido referidas por otros autores.

Las variables numéricas sin significancia estadística fueron: edad, peso, talla, número de hijos, visitas a la iglesia, número de veces actividad física por semana, 
minutos actividad física por día, índice de masa corporal, número de cigarrillos consumidos al día, número de bebidas alcohólicas consumidas por semana, tiempo para llegar al trabajo, tiempo de vida en pareja, tiempo de vida como viuda, número de veces por mes que asiste a la iglesia, años de servicio en la institución y número de ausencia al trabajo por incapacidad laboral.

Las variables categóricas sin significancia estadística, fueron: sexo, raza, pertenencia a una religión, tratamiento actual para nervios, tomar medicamentos para dormir, comer bien, realizar actividad física, consumir tabaco, consumir alcohol, el lugar de trabajo dentro de la institución, régimen de afiliación en salud y índice de masa corporal.

Las variables categóricas y cuantitativas, con significación estadística incluidas en el análisis multivariado, fueron: sentirse mal al ingreso a la institución, sentirse mal actualmente, número de horas de sueño por día, trabajar más de 48 horas por semana, estado civil, años de trabajo en la institución, horas de sueño día.

El test de Hosmer-Lemeshow arrojó un $\mathrm{Chi}^{2}=1,77$, $\mathrm{gl}=3, p=0,18$, indicando buen ajuste del modelo logístico a los datos disponibles. El poder global de clasificación correcto del modelo fue de 65,5\%, siendo $55,1 \%$ para los casos (Sensibilidad) y $75 \%$ para los controles (Especificidad).

La regresión logística mostró dos variables que aumentan la probabilidad de desarrollar el SDL: el trabajar más de 48 horas por semana y no sentirse bien con el trabajo realizado. Mientras que dormir más de 7 horas diariamente, tiene un efecto protector, es decir, cuando una auxiliar duerme lo suficiente diariamente, tendrá, en promedio, un 30\% menos de probabilidad de desarrollar el SDL y esta probabilidad se mueve en un rango comprendido entre el 9\% y el $47 \%$ (Tabla 4 ).

El modelo se ajustó por otras variables, entre ellas, la edad, la raza/etnia, el estado civil, asistir a la iglesia, el tiempo en llegar al lugar de trabajo, el comer bien, hacer ejercicio, consumir tabaco y licor, número de hijos, años de servicio en la institución, ingesta de medicamentos para los "nervios" o para dormir y otras más. La variable sexo no fue incluida en el modelo ya que los hombres representan sólo el 4,9\%, arrojando una casi perfecta separación. El análisis exploratorio mostró una distribución cuadrática en la relación entre la edad y los años de trabajo en la institución, en las auxiliares de enfermería que fueron clasificadas con el SDL.

Tabla 4. Factores asociados con el SDL. Regresión logística

\begin{tabular}{|c|c|c|c|}
\hline \multirow[b]{2}{*}{ Factores Asociados } & \multirow[b]{2}{*}{$O R$} & \multicolumn{2}{|c|}{ IC 95\% } \\
\hline & & $\begin{array}{l}\text { Límite } \\
\text { inferior }\end{array}$ & $\begin{array}{l}\text { Límite } \\
\text { superior }\end{array}$ \\
\hline $\begin{array}{l}\text { Horas trabajadas por semana } \\
\text { (Base }=48 \text { horas o menos) }\end{array}$ & 1,02 & 1,01 & 1,05 \\
\hline $\begin{array}{l}\text { Sentirse mal actualmente en } \\
\text { el trabajo (Base=sentirse } \\
\text { bien) }\end{array}$ & 3,87 & 1,18 & 10,71 \\
\hline $\begin{array}{l}\text { Horas sueño por día (Base= } 7 \\
\text { o más) }\end{array}$ & 0,70 & 0,53 & 0,91 \\
\hline
\end{tabular}

\section{Discusión}

El lugar de trabajo es muy importante para el bienestar de los trabajadores, ya que en promedio se permanece casi la mitad de las horas de vigilia en ese lugar $\mathrm{y}$ las condiciones de trabajo son un determinante para la salud. A menudo allí se enfrentan situaciones estresantes y cuando la tensión es permanente la capacidad de resiliencia de los trabajadores puede verse afectada, trayendo como consecuencia el agotamiento físico y emocional, lo cual compromete la calidad de vida y capacidad para proporcionar el mejor cuidado clínico(16,17).

Los factores generadores de estrés en el ámbito laboral de los trabajadores sanitarios, son múltiples y variados, algunos de ellos aumentan el riesgo de desarrollar tensión y agotamiento, entre los cuales se han destacado, el aumento de la carga de trabajo, el horario de trabajo extendido, la respuesta emocional al contacto con pacientes que sufren o que están muriendo, y los problemas y conflictos de la organización (Tabla 2) ${ }^{(18,19)}$.

Los servicios con la mayor prevalencia del SDL son: urgencias con $26,7 \%$, cirugía con $20,5 \%$ y medicina interna con $8,7 \%$. La prevalencia según los componentes, en este estudio, es $11,8 \%$ para Agotamiento Emocional (AE), 14,9\% para Despersonalización (DP) y 47,8\% para Realización Personal (RP). (Tabla 3). La prevalencia total del SDL es $44,7 \%$, inferior a la reportada por el estudio multicéntrico realizado en Europa(20) cuya prevalencia es $54,7 \%$. 
Un estudio realizado en Bogotá reportó el Agotamiento Emocional como indicador de estrés, en $46 \%$ de las auxiliares de enfermería; desglosado en un 33\% de ellas con un nivel bajo, el 33\% con nivel medio y el $21 \%$ con nivel alto. Para el componente de Despersonalización, 33\% nivel bajo; 37\% medio y $30 \%$ alto. Para la Realización Personal, 67\% nivel bajo; $19 \%$ medio y $14 \%$ alto(21). El nivel de realización personal bajo es mayor que en el presente estudio, $47,8 \%$.

En Córdoba, España, el 66,0\% del total del personal de enfermería estudiado presentaba SDL, distribuido así: 58,5\% con Agotamiento Emocional, 59,1\% con Despersonalización y 65,3\% con poca Realización Personal(22). Como se puede observar todas estas cifras se encuentran por encima de las encontradas en este estudio.

Con relación a los factores asociados, en Francia, el insomnio estaba asociado con la ocurrencia del SDL, hallazgo similar al de este estudio OR=14,7 IC95\% $(9,8-21,7)$ y en otro estudio la calidad del sueño también influye en el desarrollo del SDL $(23,24)$.

En China, el 41,8\% de los médicos encuestados referían intención de abandonar el sitio de trabajo debido al estrés que les generaba, y aquellos que trabajaban más de 40 horas a la semana, tenían 1,4 más probabilidad de abandonarlo, debido a la misma condición(25). En el presente estudio, las auxiliares que trabajaban más de 48 horas a la semana, presentan un riesgo de 1,02 de desarrollar el SDL y este riesgo se mueve en un rango comprendido entre $1 \%$ y $5 \%$.

En Taiwán, las enfermeras eran las funcionarias del sistema sanitario con una prevalencia del SDL de $66,1 \%$ y los factores que explican el $32,6 \%$ del SL son: alto estrés laboral, exagerada demanda sobre el resultado del trabajo realizado y bajo apoyo institucional(26). En Italia, el mal sueño, similar al hallazgo de este estudio, y los días de ausencia del trabajo por cualquier enfermedad se interpretaron como signos predictivos indirectos de riesgo del SDL(27).

Un hallazgo novedoso, no encontrado en la literatura revisada, fue que la variable edad de las auxiliares de enfermería presenta un comportamiento cuadrático, sugiriendo diversidad en los factores asociados en los extremos de la distribución etaria.
A nivel internacional, las evidencias disponibles sobre intervenciones en el sistema de salud, orientadas hacia la disminución de síndrome de agotamiento laboral, aun son limitadas. En este marco, un Comité creado en Estados Unidos, para proponer lineamientos estratégicos para su prevención y control, no logró hacer recomendaciones específicas sobre intervenciones costo/efectivas. Sin embargo, el informe recomienda que las organizaciones de salud deben crear, implementar y evaluar sus propias intervenciones mediante el uso de un enfoque sistémico para reducir el desgaste laboral; utilizando métodos rigurosos para evaluar el síndrome y sus riesgos, así como compartir las lecciones aprendidas con otros servicios de salud. El informe también recomienda invertir en investigación sobre intervención organizacional y estructural(28).

Comparar los resultados de un estudio con otros no es una tarea fácil debido, fundamentalmente, a que no siempre se utiliza la misma definición de $\operatorname{caso}^{(29)}$ y, en la mayoría de los proyectos revisados, el plan de análisis llega, en el mejor de los casos, al nivel bivariado y se sabe por las evidencias disponibles, que los resultados del análisis con sólo dos variables suelen ser diferentes cuando se toman en cuenta un conjunto de variables, aun así, se hicieron algunas comparaciones, tomando en cuenta estas limitaciones(29-32). De hecho, el análisis bivariado arrojó 4 variables estadísticamente significativas y el multivariado sólo tres de ellas.

\section{Conclusiones}

La gestión del conocimiento producido en este estudio, si aplicado correctamente, beneficiara a las próximas generaciones de auxiliares de enfermería.

Las informaciones derivadas de estudios como este pueden ayudar a tomar decisiones innovadoras sobre cómo abordar el agotamiento laboral de las auxiliares de enfermería, para evitar efectos negativos sobre la calidad de los servicios prestados.

Si bien estos hallazgos representan una pieza de información sobre el complejo problema del SDL entre auxiliares de enfermería; será necesario la realización de otras investigaciones relacionadas con este tema para aumentar el cuerpo de conocimientos que permita contar con las mejores evidencias posibles para modificar este evento que afecta, de 
manera importante, a los trabajadores de la salud, en este caso a las auxiliares de enfermería.

\section{Potenciales limitaciones}

La presencia de sesgos que afecte la validez de un estudio observacional es un fenómeno omnipresente, al cual se debe prestar especial atención para su identificación y control, ya sea durante el diseño del estudio o el análisis.

El sesgo de participación e información es mínimo, debido a que la tasa de respuesta de las encuestas alcanzó el 98,9\%. Para el análisis estadístico fueron tenidas en cuentas las variables que arrojaron una tasa de respuesta igual o superior al $85 \%$.

Aun así, se considera que debe existir un sesgo residual, buena parte debido a efecto de Hawthorne que no se pudo controlar del todo, dado que las encuestas se hacían dentro de la institución, auto diligenciadas, pero en presencia de la encuestadora, igual se considera la posibilidad del sesgo de memoria.

Otra potencial fuente de sesgo está en la clasificación de los casos con el SDL, la regresión logística arrojó que la probabilidad de clasificar correctamente un caso no iba más allá del $55,1 \%$, relativamente baja, pero eso era de esperarse ya que se sabe que de los tres componentes que describen al SDL, el de la pobre realización personal es el que tienen la menor sensibilidad, tanto que algunos autores han recomendado caracterizar el SDL utilizando sólo los otros dos componentes, aún persiste una discusión metodológica al respecto.

Este estudio arrojó prevalencias menores a las reportadas por algunos estudios y superiores a otros, estas diferencias podrían explicarse por diversidad en las definiciones utilizadas y en los puntos de corte para la clasificación de los casos, además, de la variación aleatoria usualmente encontrada en este tipo de estudios.

\section{Agradecimientos}

A todas las auxiliares que consintieron participar en la investigación, a los funcionarios de la institución que apoyaron la realización de este estudio y los colegas del Grupo de Gestión del Conocimiento e
Innovación (GECOI), por su decidida participación en la recolección de los datos.

Conflicto de intereses: Ninguno declarado por los autores.

\section{Referencias}

1. Organizacion Internacional del Trabajo. Estres en el trabajo: Un reto colectivo. Servicio de administración del trabajo, Inspección del trabajo y seguridad y Salud en el trabajo. Ginebra: Organización Internacional del Trabajo (OIT); 2016. 62 p. ISBN 978-92-2-330641-0

2. Bailey DS. Burnout harms workers' physical health through many pathways. [Internet]. American Psychological Association. 2006[cited 2018 Jul 3];37(7):11. Available from: http://www.apa.org/monitor/jun06/burnout

3. Nguyen NS, Métraux EL, Morris-Singer AF. Combating Clinician Burnout with Community-Building. NEJM Catal Innov Care Deliv. 2018. Available from: https://catalyst.nejm.org/doi/full/10.1056/CAT.18.0124

4. Adriaenssens J, De Gucht V, Maes S. Determinants and prevalence of burnout in emergency nurses: A systematic review of 25 years of research. Int J Nurs Stud. 2015;52(2):649-61. doi: 10.1016/j.ijnurstu.2014.11.004.

5. Cañadas-De la Fuente GA, Vargas C, San Luis C, García I, Cañadas GR, De la Fuente EI. Risk factors and prevalence of burnout syndrome in the nursing profession. Int J Nurs Stud. 2015;52(1):240-9. doi: 10.1016/j.ijnurstu.2014.07.001.

6. Vargas C, Cañadas GA, Aguayo R, Fernández R, De la Fuente EI. Which occupational risk factors are associated with burnout in nursing? A meta-analytic study. Int J Clin Heal Psychol [Internet]. 2014;14(1):28-38. Available from: http://dx.doi.org/10.1016/S1697-2600(14)70034-1

7. EU-OSHA. Calculating the cost of work-related stress and psychosocial risks [Internet]. Www.Healthy-Workplaces.Eu. 2014. 0-41 p. Available from: https://osha.europa.eu/en/publications/literature_reviews /calculating-the-cost-of-work-related-stress-andpsychosocial-risks/view

8. ICD-11 - Mortality and Morbidity Statistics [Internet]. [cited 2018 Jul 1]. Available from: https://icd.who.int/browse11/l$\mathrm{m} / \mathrm{en}$

9. Schaufeli W, De Witte H, Desart S. Burnout Assessment Tool (BAT)-Development, Validity, and Reliability. Int. J. Environ. Res. Public Health. 2020;17(24):9495. doi:10.3390/ijerph17249495.

10. Lee H-F, Kuo H-T, Chang C-L, Hsu C-C, Chien T-W. Determining Cutting Points of the Maslach Burnout Inventory for Nurses to Measure Their Level of Burnout Online. Hist Res [Internet]. 2017;5(1):1-8. Available from: https://doi.org/10.11648/j.history.20170501.11.

11. Maslach C, Jackson SE. The measurement of experienced burnout. J Organ Behav. 1981;2(2):99-113. doi: 10.1002/job.4030020205.

12. Córdoba L, Tamayo JA, González MA, Martínez MI, Rosales A, Barbato SH. Adaptation and validation of the Maslach Burnout Inventory-Human Services Survey in Cali, Colombia. Colomb Med. 2011;42(3):286-93. Available from: https://doi.org/10.25100/cm.v42i3.874 
13. IBM Corp. IBM SPSS Statistics for Windows. Version 24.0. Armonk, NY: IBM Corp; 2016. Available from: https://www.ibm.com/support/pages/how-cite-ibm-spssstatistics-or-earlier-versions-spss

14. Hosmer D, Lemeshow S. Applied Logistic Regression. New York: John Wiley \& Sons; 1989. ISBN 0-471-61553-6. Available from: http://resource.heartonline.cn/20150528/1_3k0QSTg.pdf

15. Kleinbaum DG. Logistic regression: A self-Learning Text. Statistics in the Health Sciences. New York: Springer; 1992. ISBN 0-387-941428

16. Rippstein-Leuenberger $K$, Mauthner 0 , Sexton JB, Schwendimann R. A qualitative analysis of the Three Good Things intervention in healthcare workers. BMJ Open. 2017;7(5):e015826. doi: 10.1136/bmjopen-2017-015826.

17. Innstrand ST, Christensen M. The Workplace as a setting: Health Promotion in the Workplace: Siw Tone Innstrand. Eur J Public Health [Internet]. 2015;25(Suppl 3). Available from: https://doi.org/10.1093/eurpub/ckv169.067

18. Ladher N. Shining a light on burnout. BMJ. 2017;358:3609. Available from: https://doi.org/10.1136/bmj.j3609

19. Saborío Morales L, Hidalgo Murillo LF. Síndrome de Burnout. Medicina Legal de Costa Rica. 2015;32(1):119-24. On-line version ISSN 2215-5287Print version ISSN 1409-0015. Available from: http://www.scielo.sa.cr/scielo.php?script=sci_arttext\&pid= S1409-00152015000100014\&lng=en

20. Dall'Ora C, Griffiths P, Ball J, Simon M, Aiken LH. Association of $12 \mathrm{~h}$ shifts and nurses' job satisfaction, burnout and intention to leave: Findings from a cross-sectional study of 12 European countries. BMJ Open. 2015;5(9):e008331. Available from: https://doi.org/10.1136/bmjopen-2015008331

21. Fajardo Zapata ÁL. Síndrome de burnout en auxiliares de enfermería. Inclusión Y Desarrollo. 2015;2(2):62-8. ISSN2389-7341. Available from: https://doi.org/10.26620/uniminuto.inclusion.2.2.2015.6268

22. Alba Martín R. Burnout en enfermería: prevalencia y factores relacionados en el medio hospitalario. Rev Científica la Soc Española Enfermería Neurológica [Internet]. 2015;41(1):914. Available

from: http://www.sciencedirect.com/science/article/pii/S20135 24615000045

23. Gomez-Garcia T, Ruzafa-Martinez M, Fuentelsaz-Gallego C, Madrid JA, Rol MA, Martinez-Madrid MJ, et al. Nurses' sleep quality, work environment and quality of care in the Spanish
National Health System: Observational study among different shifts. BMJ Open. 2016;6(8):e012073. doi: 10.1136/bmjopen-2016-012073.

24. Metlaine A, Sauvet F, Gomez-Merino D, Elbaz M, Delafosse JY, Leger D, et al. Association between insomnia symptoms, job strain and burnout syndrome: A cross-sectional survey of 1300 financial workers. BMJ Open. 2017;7(1):e012816. Available from: https://doi.org/10.1136/bmjopen-2016012816

25. Gómez-García T, Ruzafa-Martínez M, Fuentelsaz-Gallego C, et al. Nurses' sleep quality, work environment, and quality of care in the Spanish National Health System: observational study among different shifts. BMJ Open 2016;6:e012073. doi: 10.1136/bmjopen-2016-012073

26. Lu Y, Hu XM, Huang XL, Zhuang XD, Guo P, Feng LF, et al. The relationship between job satisfaction, work stress, workfamily conflict, and turnover intention among physicians in Guangdong, China: A cross-sectional study. BMJ Open. 2017;7(5):e014894. doi:10.1136/bmjopen-2016-014894.

27. Chou LP, Li CY, Hu SC. Job stress and burnout in hospital employees: Comparisons of different medical professions in a regional hospital in Taiwan. BMJ Open. 2014;4(2):e004185. doi: 10.1136/bmjopen-2013-004185.

28. Hinnenthal IM, Anselmo R, Delfino E, Messina G, Ricciardi W, Vaccaro D, et al. Burn-out and correlated factors of nurses and health care workers in 23 nursing homes in Liguria (Italy): Ina Maria Hinnenthal. Eur J Public Health. 2014;24(Suppl 2). doi:10.1093/eurpub/cku166.114.

29. National Academies of Sciences, Engineering, and Medicine. Taking Action Against Clinician Burnout: A Systems Approach to Professional Well-Being [Internet]. Washington, DC: The National Academies Press; 2019. Available from: https://www.nap.edu/catalog/25521/taking-actionagainst-clinician-burnout-a-systems-approach-toprofessional

30. Bergonzoli G. Epidemiologia y Estadistica: 1001 preguntas. lo que no se atrevio preguntar, pero siempre quiso saber. Cimpretics. Santiago de Calli, 2019. ISBN 978-958-48-60590

31. Kleinbaum DG, Kupper LL, Muller KE, Nizam A. Applied Regression Analysis and Other Multivariable Methods. 1988. Wadsworth Publishing Company. ISBN 0-97150-123-6

32. Rencher AC. Methods of Multivariate Analysis. Second Edition. John Willey \& Sons, editor. New York: Inc. Publication; 2002. ISBN: 978-0-471-46172-2. Available from: https://www.ipen.br/biblioteca/slr/cel/0241 